\title{
MicroRNA-135b-5p prevents oxygen-glucose deprivation and reoxygenation-induced neuronal injury through regulation of the GSK-3 $\beta /$ Nrf2/ARE signaling pathway
}

Qiang Duan, Wei Sun, Hua Yuan, Xiang Mu

Department of Rehabilitation Medicine, Xijing Hospital, The Fourth Military Medical University, Xi'an, China

Submitted: 27 July 2017

Accepted: 30 August 2017

Arch Med Sci 2018; 14, 4: 735-744

DOI: https://doi.org/10.5114/aoms.2017.71076

Copyright $\odot 2017$ Termedia \& Banach

\section{Abstract}

Introduction: MicroRNAs (miRNAs) are emerging as critical regulators in the pathological process of cerebral ischemia/reperfusion injury. miRNAs play an important role in regulating neuronal survival. miR-135b-5p has been reported as an important miRNA in regulating cell apoptosis. However, the role of miR-135b-5p in regulating neuronal survival remains poorly understood. Here, we aimed to investigate the role of miR-135b-5p in cerebral ischemia/ reperfusion using an in vitro model of oxygen-glucose deprivation and reoxygenation-(OGD/R) induced neuron injury.

Material and methods: miRNA, mRNA and protein expression was detected by real-time quantitative polymerase chain reaction and Western blot. Cell viability was detected by cell counting kit- 8 and lactate dehydrogenase assays. Cell apoptosis was detected by caspase- 3 activity assay. Oxidative stress was determined using commercial kits. The target of miR-135b-5p was confirmed by dual-luciferase reporter assay.

Results: We found that miR-135b-5p expression was significantly decreased in hippocampal neurons receiving $O G D / R$ treatment. Overexpression of miR135b-5p markedly alleviated OGD/R-induced cell injury and oxidative stress, whereas suppression of miR-135b-5p showed the opposite effects. We observed that miR-135b-5p directly targeted the $3^{\prime}$-untranslated region of glycogen synthase kinase- $3 \beta$ (GSK-3 $\beta$ ). We found that miR-135b-5p negatively regulates the expression of GSK-3 $\beta$ in hippocampal neurons. Moreover, miR-135b-5p overexpression promotes activation of nuclear factor erythroid 2-related factor 2 (Nrf2)/antioxidant response element (ARE) signaling. However, the restoration of GSK-3 $\beta$ expression significantly reversed the protective effects of miR-135b-5p overexpression.

Conclusions: Overall, our results suggest that miR-135b-5p protects neurons against OGD/R-induced injury through downregulation of GSK-3 $\beta$ and promotion of the Nrf2/ARE signaling pathway-mediated antioxidant responses.

Key words: GSK-3 $\beta$, ischemia/reperfusion injury, miR-135b-5p, Nrf2.

\section{Introduction}

Cerebral ischemia/reperfusion injury is a major cause of death and disability causing a heavy financial burden worldwide [1]. Insufficient blood flow to the brain causes serious brain damage, which is aggravated by blood reperfusion [2]. Ischemia/reperfusion induces calcium overload, inflammation, glutamate excitotoxicity, excessive generation

\author{
Corresponding author: \\ Hua Yuan \\ Department of \\ Rehabilitation Medicine \\ Xijing Hospital \\ The Fourth Military \\ Medical University \\ 127 Changle West Road \\ Xi'an 710032, China \\ E-mail: yuanhuaxj@163.com
}


of reactive oxygen species (ROS), and apoptosis, thus contributing to neuronal damage [3]. Thrombolytic therapy has been applied for the treatment of cerebral ischemia/reperfusion injury in clinics. However, the treatment effect is limited. Therefore, it is important to develop novel therapeutic strategies for the treatment of cerebral ischemia/ reperfusion injury.

MicroRNAs (miRNAs) are a subset of small, noncoding RNAs that can negatively regulate gene expression by binding to the $3^{\prime}$-untranslated region (UTR) of mRNA (mRNA), inhibiting translation [4]. Therefore, miRNAs are involved in regulating many biological processes, including cell proliferation, apoptosis, and survival [5]. Increasing evidence has suggested that miRNAs are involved in the pathophysiological process of cerebral ischemia/ reperfusion injury [6]. Various miRNAs are dysreg ulated during cerebral ischemia/reperfusion injury, and targeting specific miRNAs has been shown to prevent neuronal damage in vitro and in vivo [7, 8]. Therefore, miRNAs are potential and promising targets for treatment of cerebral ischemia/reperfusion injury.

Glycogen synthase kinase- $3 \beta$ (GSK-3 $\beta$ ) is a serine/threonine protein kinase that plays an important role in many biological activities, including glycogen metabolism, inflammation, embryogenesis, and neuroplasticity [9]. GSK-3 $\beta$ is abundantly expressed in neurons in the central nervous system [10]. Studies have shown that GSK-3 $\beta$ is involved in the regulation of psychiatric and neurodegenerative disorders [11]. A growing body of evidence has shown that GSK-3 $\beta$ plays a critical role in cerebral ischemia/reperfusion injury $[12,13]$. Inhibition of GSK-3 $\beta$ attenuates neuronal death and protects neurons against cerebral ischemia/ reperfusion injury [14]. Nuclear factor-E2-related factor 2 (Nrf2), a redox-sensitive transcription factor [15], has been suggested as the downstream target of GSK-3 $\beta$ [16]. Nrf2 can bind to the antioxidant response elements (ARE) in the nucleus and activate the expression of antioxidant genes, such as heme oxygenase-1 (HO-1) and NADPH-quinone oxidoreductase 1 (NQO1) [17]. Activation of Nrf2/ ARE signaling attenuates the production of ROS and neuron apoptosis, thus having a protective effect during cerebral ischemia/reperfusion injury [18]. GSK-3 $\beta$ impedes the nuclear translocation of Nrf2 and inhibits the antioxidant cell response [19]. Inhibition of GSK-3 $\beta$ prevents the neuronal damage induced by brain ischemia by activation of Nrf2/ARE signaling [20]. Therefore, targeting GSK-3 $\beta$ may lead to the discovery of novel therapeutic strategies for cerebral ischemia/reperfusion injury.

miR-135b-5p has been reported as an anti-apoptotic miRNA $[21,22]$. A recent study has shown that miR-135b-5p has a neuroprotective effect in Alzheimer's disease [23]. However, little is known about the role of miR-135b-5p in regulating neuronal survival during cerebral ischemia/ reperfusion injury. Here, we aimed to investigate the functional significance of miR-135b-5p in regulating neuron survival using an OGD/R cellular model. We found that miR-135b-5p expression was significantly decreased in hippocampal neurons receiving $\mathrm{OGD} / \mathrm{R}$ treatment. Overexpression of miR-135b-5p markedly alleviated OGD/R-induced injury. GSK-3 $\beta$ was identified as a target gene of miR-135b-5p. Moreover, overexpression of miR-135b-5p inhibited GSK-3 $\beta$ and promoted activation of the Nrf2/ARE signaling pathway. The restoration of GSK-3 $\beta$ expression significantly reversed the protective effects of miR-135b-5p overexpression. Taken together, our study suggests that miR-135b-5p protects neurons against OGD/R-induced injury through downregulation of GSK-3 $\beta$ and promotion of the Nrf2/ARE signaling pathway-mediated antioxidant responses.

\section{Material and methods}

\section{Cell culture}

The mouse hippocampal neuronal HT22 cell line was purchased from JENNIO Biological Technology (Guangzhou, China). HT22 cells were cultured in Dulbecco's Modified Eagle's Medium (DMEM; Gibco, Rockville, MD, USA) supplemented with $10 \%$ fetal bovine serum (FBS), $4.5 \mathrm{~g} / \mathrm{l}$ glucose and $1 \%$ penicillin/streptomycin mix (Sigma, St. Louis, MO, USA). Cells were cultured in a humidified incubator with $5 \% \mathrm{CO}_{2}$ at $37^{\circ} \mathrm{C}$.

\section{Induction of oxygen-glucose deprivation and reoxygenation (OGD/R) injury}

Cells were cultured in glucose-free Hank's Balanced Salt Solution and incubated in a hypoxia incubation chamber (Thermo Fisher Scientific, Shanghai, China) containing $1 \% \mathrm{O}_{2}, 5 \% \mathrm{CO}_{2}, 94 \%$ $\mathrm{N}_{2}$ at $37^{\circ} \mathrm{C}$. After culture for $6 \mathrm{~h}$, the medium was replaced with growth medium containing $4.5 \mathrm{~g} / \mathrm{l}$ glucose and cultured under normal conditions for $24 \mathrm{~h}$ reoxygenation. Cells without OGD/R treatment were used as a control.

\section{RNA isolation and real-time quantitative polymerase chain reaction (RT-qPCR)}

Total RNA was isolated using Trizol reagent (Invitrogen, Carlsbad, CA, USA) according to the manufacturer's instructions. RNA was reverse-transcribed into cDNA using the miScript Reverse Transcription Kit (Qiagen, Dusseldorf, Germany) or M-MLV Reverse Transcriptase (TaKaRa, Dalian, China). $P C R$ reactions were performed using SYBR Green 
PCR Master Mix (Applied Biosystems, Foster City, CA, USA) with the ABI7500 real-time PCR system (Applied Biosystems). U6 was used as an internal control for normalization of miR-135b-5p. $\beta$-actin was used as an internal control for normalization of GSK-3 $\beta, \mathrm{HO}-1$, and NQO1. The primer sequences were as follows: miR-135b-5p F: 5'-GGTATGGCTTTTCATTCCT-3' and R: 5'-CAGTGCGTGTCGTGGAGT3'; U6 F: 5'-ATTGGAACGATACAGAGAAGATT-3' and R: 5'-GGAACGCTTCACGAATTTG-3'; GSK-3 $\beta$ F: 5'-ATGGCAGCAAGGTAACCACAG-3' and R: 5'-TCTCGGTTCTTAAATCGCTTGTC-3'; HO-1 F: 5'-CCCAAAACTGGCCTGTAAAA-3' and R: 5'-CGTGGTCAGTCAACATGGAT-3'; NQO1 F: 5'- AGGATGGGAGGTACTCGAATC-3' and R: $5^{\prime}$ - AGGCGTCCTTCCTTATATGCTA- $3^{\prime} ; \beta$-actin F: 5'-CTGTCCCTGTATGCCTCTG-3' and R: 5'-ATGTCACGCACGATTTCC-3'. The relative gene expression was analyzed by the $2^{-\Delta \Delta C t}$ method.

\section{Cell transfection}

miR-135b-5p mimics, miR-135b-5p inhibitor, and negative control (NC) were purchased from Ambion (Austin, TX, USA). The pcDNA3.1/GSK$3 \beta$ vector was generated by inserting the open reading frame of GSK-3 $\beta$ without its $3^{\prime}$-UTR into the pcDNA3.1 vector (Sangon Biotech, Shanghai, China). Cell transfection was performed using Lipofectamine 2000 (Invitrogen) in accordance with the manufacturer's instructions.

\section{Cell viability assay}

Cell viability was detected by cell counting kit-8 (CCK-8) assay. Briefly, cells were plated into 96-well plates at a density of $5 \times 10^{3}$ cells/well and cultured overnight at $37^{\circ} \mathrm{C}$. After treatment, cells were treated with CCK-8 solution $(10 \mu \mathrm{l}$ well; Sigma) and incubated for $2 \mathrm{~h}$ at $37^{\circ} \mathrm{C}$. The absorbance value at a wavelength of $490 \mathrm{~nm}$ was determined using a microplate reader (Bio-Tek, Winooski, VT, USA).

\section{Lactate dehydrogenase (LDH) assay}

Cell injury was measured using an LDH cytotoxicity assay kit (Roche Applied Science, Indianapolis, IN, USA). Briefly, cells were lysed and treated with $\mathrm{NADH}$ and pyruvate for $15 \mathrm{~min}$ at $37^{\circ} \mathrm{C}$. The absorbance value at a wavelength of $530 \mathrm{~nm}$ was measured by a microplate reader (Bio-Tek).

\section{Caspase-3 activity assay}

Cell apoptosis was measured using a caspase-3 activity assay kit (Beyotime Biotechnology, Haimen, China). Briefly, cells were lysed and incubated with 2 mM Ac-DEVD-pNA in reaction buffer for $1-4 \mathrm{~h}$ at $37^{\circ} \mathrm{C}$. Once the color had obviously changed, the absorbance value at a wavelength of
$405 \mathrm{~nm}$ was detected using a microplate reader (Bio-Tek).

\section{ROS measurement}

Reactive oxide species levels were measured using a dichlorofluorescein diacetate (DCFH-DA) assay. Briefly, cells were incubated with $50 \mu \mathrm{M}$ DCFH-DA (Sigma) for $30 \mathrm{~min}$ at $37^{\circ} \mathrm{C}$. Then, cells were washed, and the fluorescence intensity was measured by a fluorescence spectrophotometer (Bio-Tek) with an excitation wavelength of $485 \mathrm{~nm}$ and an emission wavelength of $530 \mathrm{~nm}$.

\section{Superoxide dismutase (SOD) and malondialdehyde (MDA) measurement}

The levels of SOD and MDA were evaluated using commercial kits (Nanjing Jiancheng Bioengineering Institute, Nanjing, China) in accordance with the manufacturer's protocols.

\section{Dual-luciferase reporter assay}

The fragments of wild-type or mutant GSK$3 \beta$ 3'-UTR containing the predicted binding site of miR-135b-5p was inserted into the pmirGLO vector (Promega, Madison, WI, USA). HT22 cells were cotransfected with a pmirGLO vector containing wild-type or mutant GSK-3 3 3'-UTR and miR-135b-5p mimics or inhibitor using Lipofectamine 2000 (Invitrogen). After culturing for 48 h, cells were lysed, and the luciferase activity was measured using the Dual-Luciferase Reporter Assay System (Promega). For measurement of ARE activity, HT22 cells were cotransfected with ARE reporter vector (Promega), phRL-TK Renilla luciferase vector (Promega) and miR-135b-5p mimics or inhibitor. After culturing for $48 \mathrm{~h}$, the luciferase activity was measured using the Dual-Luciferase Reporter Assay System (Promega).

\section{Western blot analysis}

Equivalent amounts $(40 \mu \mathrm{g})$ of proteins from each sample were fractionated by $10 \%$ sodium dodecyl sulfate polyacrylamide gels. The separated proteins were then electroblotted onto a polyvinylidene fluoride membrane (Millipore, Billerica, MA, USA). After blocking with $5 \%$ non-fat milk, the membrane was incubated with anti-GSK-3 $\beta$ (Cell Signaling Technology, Danvers, MA, USA), anti-Nrf2, and anti- $\beta$-actin (Abcam, Cambridge, UK) overnight at $4^{\circ} \mathrm{C}$. Afterwards, the membrane was probed with horseradish peroxidase-conjugated secondary antibodies (1: 5000; Sigma) for $1 \mathrm{~h}$ at room temperature. Protein bands were visualized with the chemiluminescent reagents (Millipore). Band density was analyzed by Image-Pro Plus 6.0 software. $\beta$-actin served as the loading control for 
normalization of GSK-3 $\beta$ and Nrf2 protein expression.

\section{Statistical analysis}

Data were expressed as mean \pm standard deviation. Student's $t$-test and one-way analysis of variance (ANOVA) with Bonferroni post hoc test were used to determine the significance levels of differences using SPSS 18.0 statistical software (SPSS Inc., Chicago, IL, USA). Differences with $p$-values less than 0.05 were considered statistically significant.

\section{Results}

\section{MiR-135b-5p is downregulated in hippocampal neurons receiving OGD/R treatment}

To investigate the potential role of miR-135b$5 p$ in cerebral ischemia/reperfusion injury, we examined the expression pattern of miR-135b-5p in hippocampal neurons receiving OGD/R treatment by RT-qPCR. We found that miR-135b-5p was significantly downregulated in hippocampal neurons receiving OGD/R treatment compared to the control group (Figure $1 \mathrm{~A}$ ), implying an important role of miR-135b-5p in hippocampal neurons receiving OGD/R treatment.

\section{Overexpression of MiR-135b-5p attenuates OGD/R-induced injury}

To investigate the precise biological function of miR-135b-5p in hippocampal neurons receiving OGD/R treatment, we detected the effect of miR-135b-5p overexpression on OGD/R-induced injury. Transfection of miR-135b-5p mimics significantly upregulated the expression of miR-135b-5p in cells receiving $O G D / R$ treatment (Figure $1 \mathrm{~B}$ ). CCK-8 assay showed that miR-135b-5p overexpres-

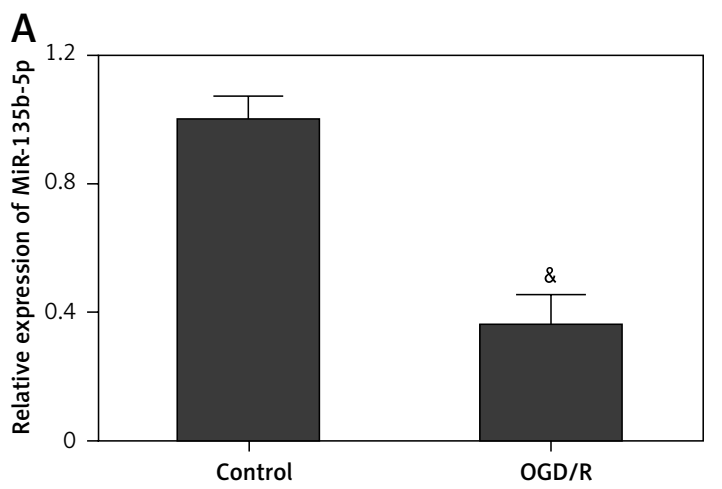

sion significantly improved the viability of hippocampal neurons receiving OGD/R treatment (Figure 2 A). The LDH assay showed that miR-135-5p overexpression markedly attenuated OGD/R-induced cell death (Figure 2 B). Furthermore, OGD/R-induced cell apoptosis was also significantly reduced by miR-135b-5p overexpression (Figure 2 C). By contrast, suppression of miR-135b-5p significantly aggravated OGD/R-induced injury in hippocampal neurons (Figures $2 \mathrm{~A}-\mathrm{C}$ ). These results suggest that miR-135b-5p attenuates OGD/R-induced injury.

\section{Overexpression of MiR-135b-5p inhibits oxidative stress induced by $O G D / R$ treatment}

To further investigate the biological function of miR-135b-5p in hippocampal neurons receiving OGD/R treatment, we detected the effect of miR-135b-5p in oxidative stress induced by OGD/R treatment. We found that overexpression of miR-135b-5p significantly reduced the ROS production induced by OGD/R treatment (Figure 3 A). Moreover, overexpression of miR-135b-5p significantly promoted the expression of SOD (Figure $3 \mathrm{~B}$ ) while decreasing the expression of MDA (Figure $3 \mathrm{C}$ ). In contrast, suppression of miR-135b$5 p$ showed the opposite effects (Figures $3 \mathrm{~A}-\mathrm{C}$ ). These results suggest that miR-135b-5p inhibits OGD/R oxidative stress.

\section{GSK-3 $\beta$ is a target gene of MIR-135b-5p}

To investigate the molecular mechanism by which miR-135b-5p regulates OGD/R-induced injury, we predicted the downstream target genes of miR-135b-5p by bioinformatics analysis. Interestingly, we found that GSK-3 $\beta$, an important gene involved in cerebral ischemia and reperfusion [12, 13], was a potential target gene of miR-135b-5p (Figures 4 A). To investigate whether miR-135b-5p

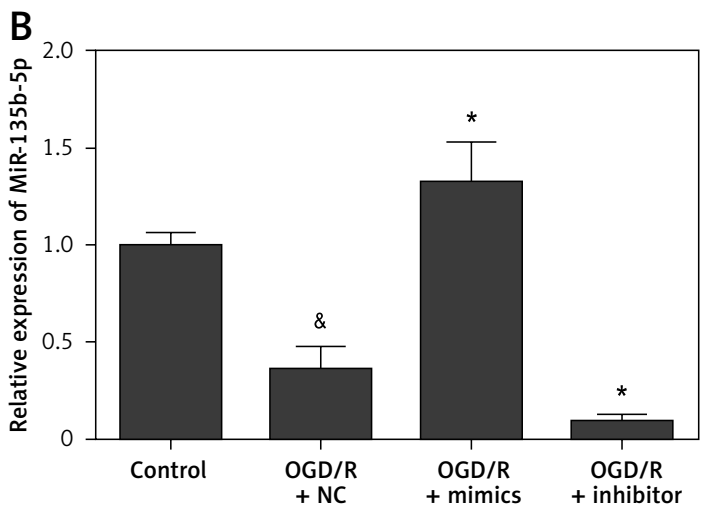

Figure 1. Expression of miR-135b-5p in hippocampal neurons receiving OGD/R treatment. A - RT-qPCR analysis of miR-135b-5p expression in HT22 cells with OGD/R treatment. Cells cultured in normal condition were used as a control. B - RT-qPCR analysis of miR-135b-5p expression in HT22 cells transfected with miR-135b-5p mimics or inhibitor for $24 \mathrm{~h}$ followed by OGD/R treatment

${ }^{*} p<0.05$ vs. control and ${ }^{*} p<0.05$ vs. OGD $/ R+N C$. 

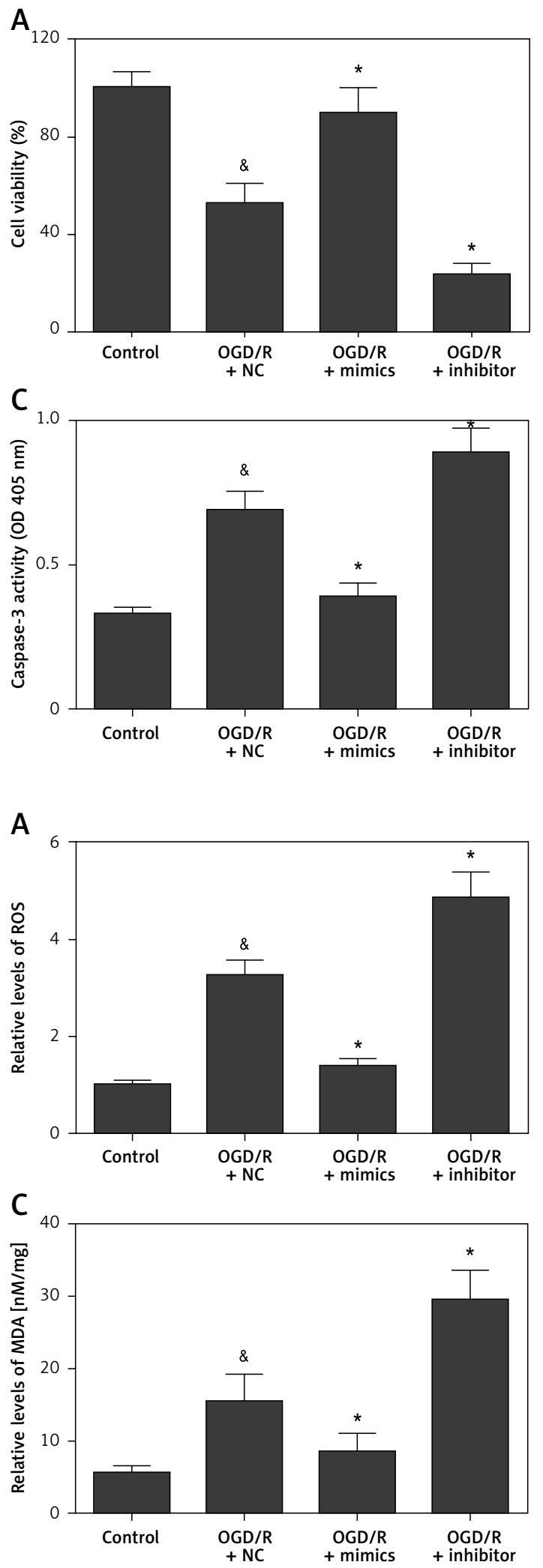

directly targeted the $3^{\prime}$-UTR of GSK-3 $\beta$, we performed dual-luciferase reporter assays. We found that overexpression of miR-135b-5p significantly decreased the luciferase activity of a reporter vector containing the wild-type GSK-3 $\beta$ 3'-UTR,

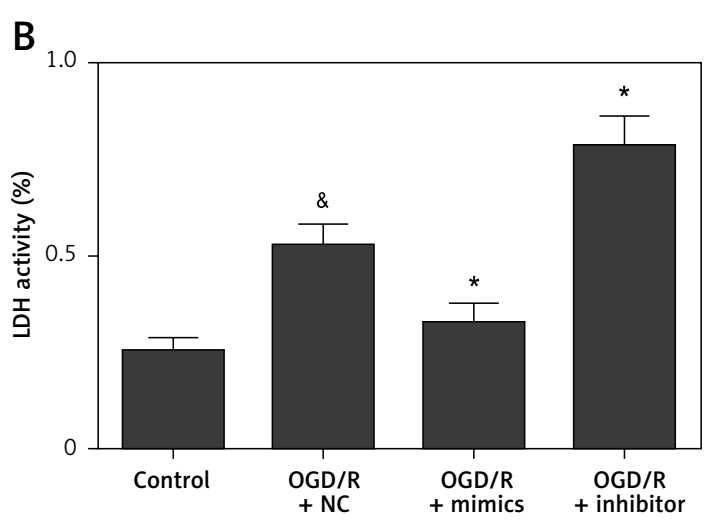

Figure 2. Overexpression of miR-135b-5p attenuates OGD/R-induced injury. HT22 cells were transfected with miR-135b-5p mimics or inhibitor for $24 \mathrm{~h}$ and then subjected to OGD/R treatment. A - Cell viability was measured by CCK-8 assay. B - Cell death was evaluated by LDH assay. C - Cell apoptosis was detected by caspase- 3 activity assay ${ }^{\&} p<0.05$ vs. control and ${ }^{*} p<0.05$ vs. OGD $/ R+N C$.

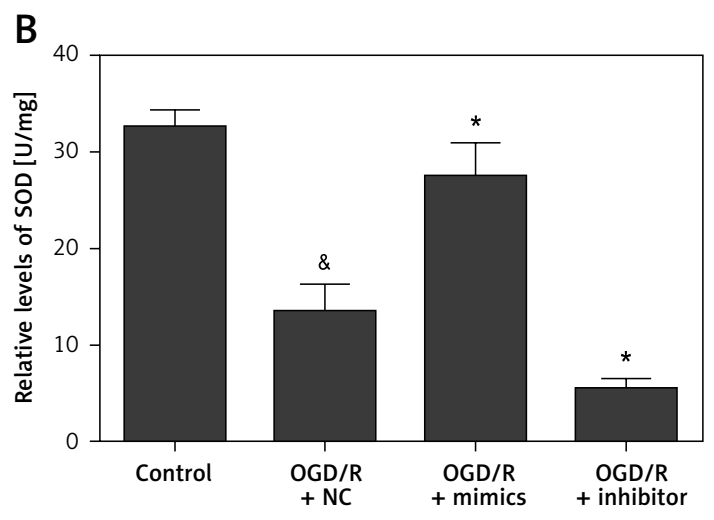

Figure 3. Overexpression of miR-135b-5p inhibits OGD/R-induced oxidative stress. HT22 cells were transfected with miR-135b-5p mimics or inhibitor for $24 \mathrm{~h}$ and then subjected to OGD/R treatment. A - ROS levels were measured by DCFH-DA assay. Levels of SOD (B) and MDA (C) were measured using commercial kits

${ }^{\&} p<0.05$ vs. control and ${ }^{*} p<0.05$ vs. OGD/R+NC.

whereas suppression of miR-135b-5p promoted luciferase activity (Figure $4 \mathrm{~B}$ ). However, miR$135 b-5 p$ showed no significant effect on the luciferase activity of the reporter vector containing the mutant GSK-3 $\beta$ 3'-UTR (Figure 4 B). To confirm 
A

5'-UAGACGAGCUUUUUAAAGCCAUA-3' wild-type GSK-3ß | || |||||

3'-AGUGUAUCCUUACUUUUCGGUAU-5' mmu-miR-135b-5p | 1 | |

5'-UAGACGAGCUUUUUAUACCGAAA-3' mutant GSK-3 $\beta$

B

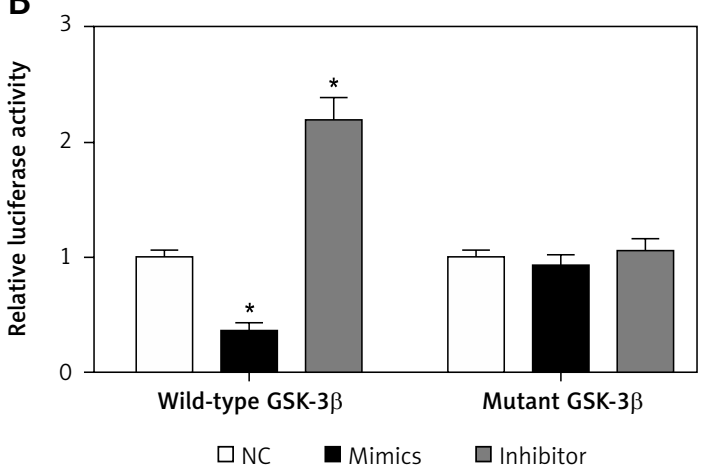

Figure 4. miR-135b-5p targets the $3^{\prime}$-UTR of GSK$3 \beta$. A - Diagram of the predicted miR-135b-5p binding site in the $3^{\prime}$-UTR of GSK-3 $\beta$. B - A dual-luciferase reporter assay was performed to examine whether miR-135b-5p directly binds to the GSK-3 $\beta$ $3^{\prime}$-UTR in HT22 cells

${ }^{*} p<0.05$ vs. NC.

that GSK-3 $\beta$ is a direct target gene of miR-135b$5 p$, we detected the effect of miR-135b-5p on GSK-3 $\beta$ expression by RT-qPCR and Western blot analysis. We found that overexpression of miR135b-5p significantly decreased the mRNA and protein expression of GSK-3 $\beta$ while suppression of miR-135b-5p promoted GSK-3 $\beta$ expression (Figures $5 \mathrm{~A}$ and $\mathrm{B}$ ). Overall, these results suggest that GSK-3 $\beta$ is a target gene of miR-135b-5p.

\section{Overexpression of Mir-135b-5p promotes activation of the Nrf2/ARE signaling pathway}

To further investigate the molecular basis of miR-135b-5p in regulating OGD/R-induced inju- ry, we detected the effect of miR-135b-5p on the Nrf2/ARE signaling pathway, the downstream pathway of GSK-3 $\beta[19,20]$. We found that overexpression of miR-135b-5p significantly promoted the expression of Nrf2 in cells with OGD/R treatment (Figure 6 A). Overexpression of miR-135b-5p also significantly increased ARE activity (Figure $6 \mathrm{~B}$ ). Furthermore, the expression of Nrf2/ARE downstream target genes, including $\mathrm{HO}-1$ (Figures $6 \mathrm{C}$ ) and NQO1 (Figure $6 \mathrm{D}$ ), was also markedly upregulated by miR-135b-5p overexpression. These results suggest that miR-135b-5p overexpression increases activation of the Nrf2/ARE signaling pathway.

\section{Restoration of GSK-3 $\beta$ expression reverses the protective effect of Mir-135b-5p overexpression}

To confirm that GSK-3 $\beta$ is a functional target gene of miR-135b-5p, we performed rescue experiments by restoration of GSK-3 $\beta$ expression in cells transfected with miR-135b-5p mimics. We found that transfection of the pcDNA3.1/GSK$3 \beta$ vector significantly restored the expression of GSK-3 $\beta$ in cells transfected with miR-135b$5 p$ mimics (Figure $7 \mathrm{~A}$ ). Moreover, restoration of GSK-3 $\beta$ expression significantly blocked the effect of miR-135b-5p overexpression on activation of the Nrf2/ARE signaling pathway (Figures 7 B-D). As expected, the protective effect of miR-135b-5p overexpression against OGD/R-induced injury was significantly abrogated by GSK-3 $\beta$ overexpression (Figures $8 \mathrm{~A}-\mathrm{C}$ ). These results suggest that GSK-3 $\beta$ is a functional target gene of $\mathrm{miR}-135 \mathrm{~b}-5 \mathrm{p}$ in regulating OGD/R-induced injury.

\section{Discussion}

Cerebral ischemia/reperfusion injury is a complex and severe pathological process that involves
A

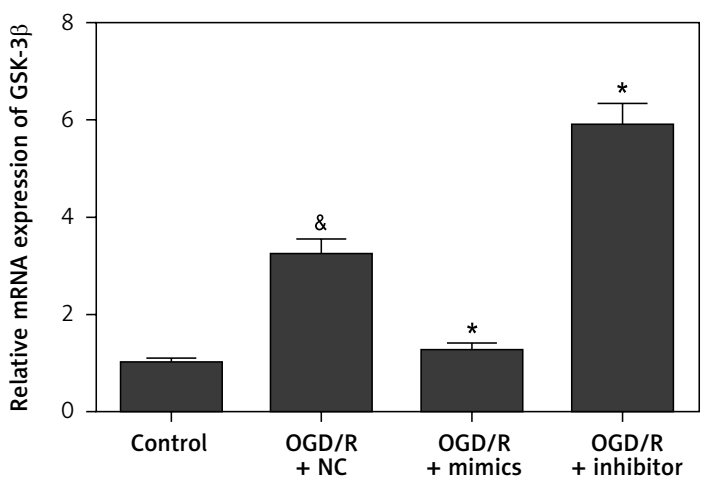

B

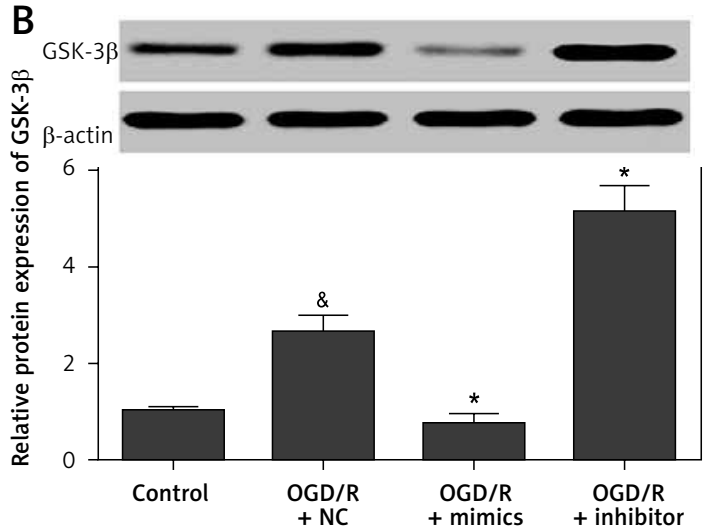

Figure 5. miR-135b-5p negatively regulates GSK-3 $\beta$ expression. HT22 cells were transfected with miR-135b-5p mimics or inhibitor for $24 \mathrm{~h}$ and then subjected to OGD/R treatment. A - The mRNA expression of GSK-3 $\beta$ was detected by RT-qPCR. B - Protein expression of GSK-3 $\beta$ was detected by Western blot

${ }^{*} p<0.05$ vs. control and ${ }^{*} p<0.05$ vs. OGD $/ R+N C$ 

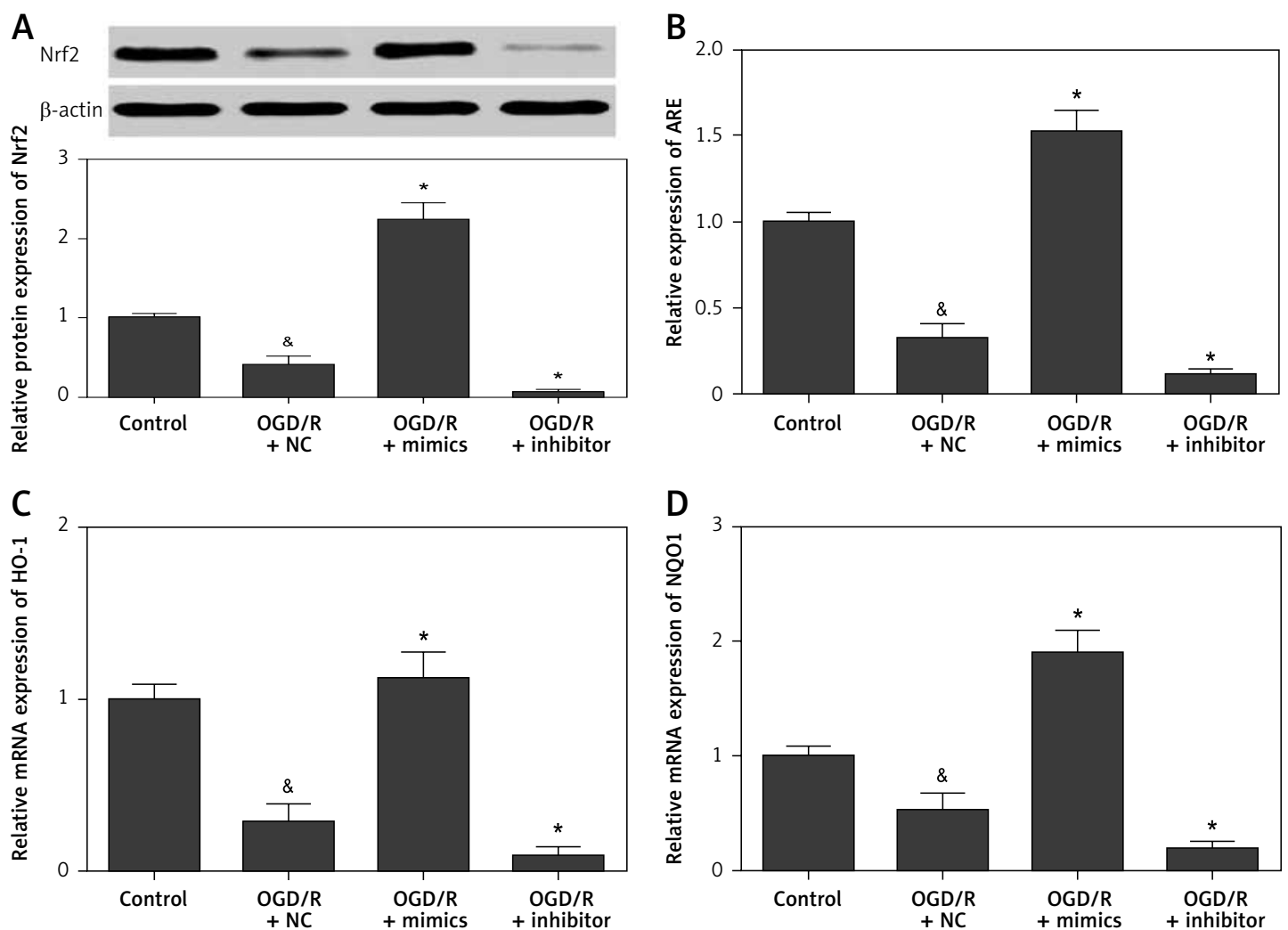

Figure 6. Overexpression of miR-135b-5p promotes activation of the Nrf2/ARE signaling pathway. HT22 cells were transfected with ARE reporter vector and miR-135b-5p mimics or inhibitor for $24 \mathrm{~h}$ and then subjected to OGD/R treatment. A - Protein expression of Nrf2 was detected by Western blot analysis. B - ARE activity was detected by dual-luciferase reporter assays. The mRNA expression of HO-1 (C) and NQO1 (D) was detected by RT-qPCR

${ }^{\star} p<0.05$ vs. control and ${ }^{*} p<0.05$ vs. OGD/R $+N C$.

inflammation, protein synthesis inhibition, and impaired mitochondrial function and energy metabolism, which cause neuron loss and brain damage [24]. Therefore, the concept of single-drug intervention cannot be effective [24]. Further experimental research is needed to gain a better understanding of the molecular pathogenesis of cerebral ischemia/reperfusion injury and to explore novel and effective therapeutic strategies. Here, we have revealed an important role for miR-135b$5 p$ in regulating OGD/R-induced injury in neurons. We found that miR-135b-5p is significantly decreased in response to OGD/R treatment. Overexpression of miR-135b-5p significantly attenuates OGD/R-induced injury. The underlying mechanism for this might involve the Nrf2/ARE defense pathway through the inhibition of GSK-3 $\beta$. Overall, our study indicates that the miR-135b-5p/GSK$3 \beta / \mathrm{Nrf2/ARE}$ signaling pathway may play an important role in regulating neuronal survival under OGD/R-induced injury.

Studies have demonstrated that miR-135b-5p is involved in regulating inflammation and cell differentiation $[25,26]$. Various studies have shown that miR-135b-5p promotes tumorigenesis, chemoresistance, and radioresistance by inhibiting cell apoptosis [21, 27, 28]. Fan et al. reported that $\mathrm{miR}-135 \mathrm{~b}-5 \mathrm{p}$ protects osteoblastic cells from dexamethasone-induced apoptosis [29]. Overexpression of miR-135b-5p has a neuroprotective effect on Alzheimer's disease by targeting the $\beta$-site APP-cleaving enzyme 1 [23]. miR-135b-5p is decreased in the hippocampus of a rat model of temporal lobe epilepsy and may be involved in the regulation of hippocampal neuron apoptosis [30]. In mouse hippocampal neurons, miR-135b-5p is deregulated under oxidative stress [31]. However, little is known about the role of miR-135b-5p in cerebral ischemia/reperfusion injury. Herein, we investigated the potential role of miR-135b-5p in regulating neuronal survival using OGD/R-induced injury, an in vitro model of cerebral ischemia/reperfusion injury. We found that miR-135b-5p was decreased in hippocampal neurons receiving OGD/R treatment. The overexpression of miR-135b-5p markedly attenuated OGD/R-induced injury, implying a neuroprotective role for $\operatorname{miR}-135 b-5 p$ in OGD/R-induced injury.

GSK-3 $\beta$ has been suggested as an important regulator in cerebral ischemia/reperfusion injury $[12,13]$. Inhibition of GSK-3 $\beta$ attenuates neuronal death and protects neurons against cerebral ischemia/reperfusion injury [14, 32, 33]. GSK-3 $\beta$ promotes cerebral ischemia/reperfusion injury 
A
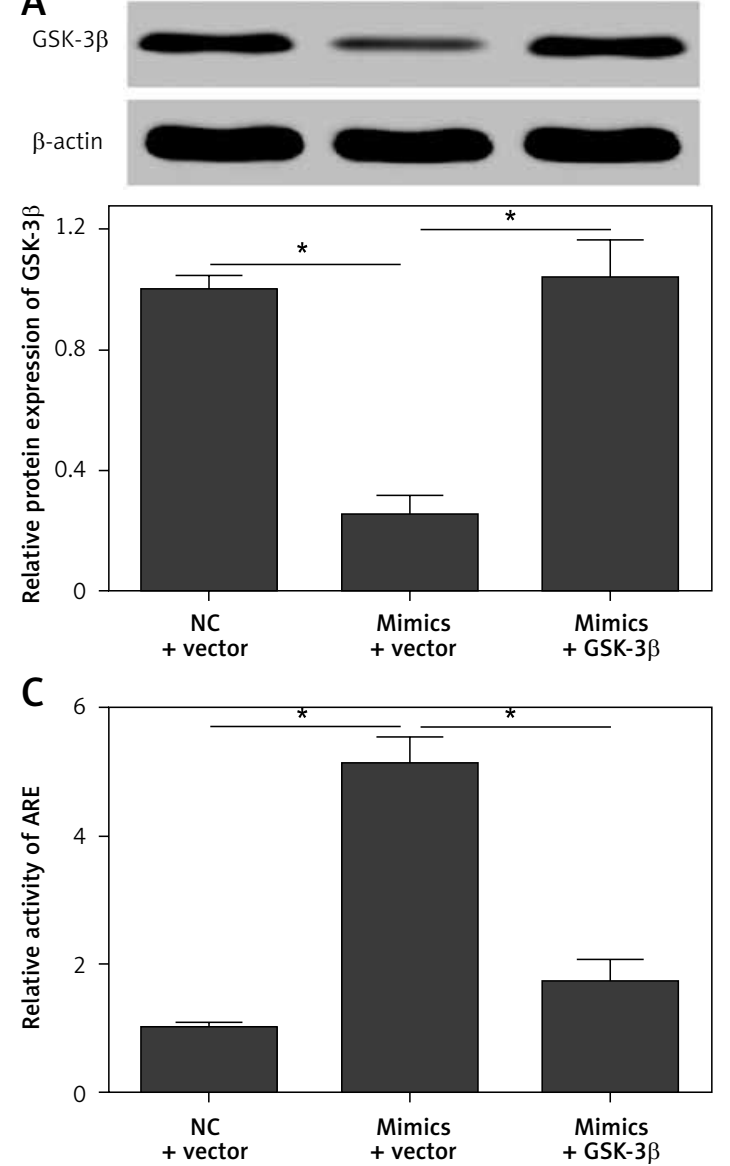

B
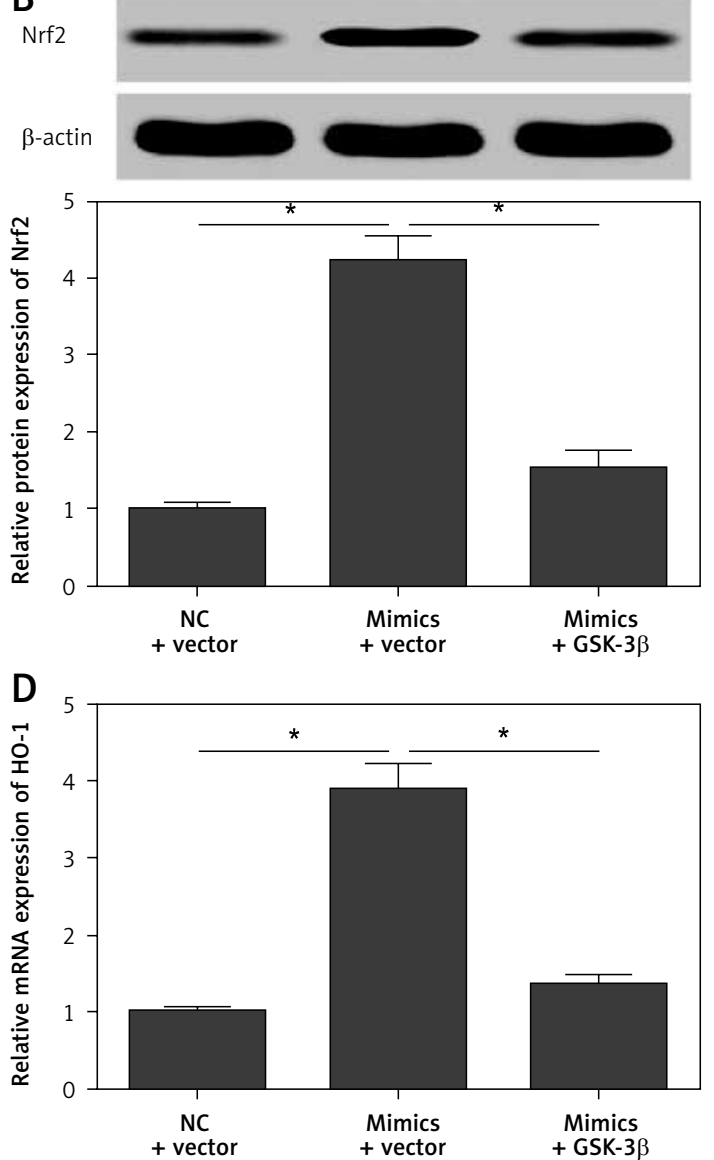

Figure 7. Restoration of GSK-3 $\beta$ expression reverses the effect of miR-135b-5p overexpression on Nrf2/ARE signaling. HT22 cells were cotransfected with pcDNA3.1/GSK-3 $\beta$ vector and miR-135b-5p mimics for $24 \mathrm{~h}$ and then subjected to OGD/R treatment. Expression of GSK-3 $\beta$ (A) and Nrf2 (B) was detected by Western blot. C - ARE activity was detected by dual-luciferase reporter assays. D - The mRNA expression of HO-1 was detected by RT-qPCR ${ }^{*} p<0.05$.

A
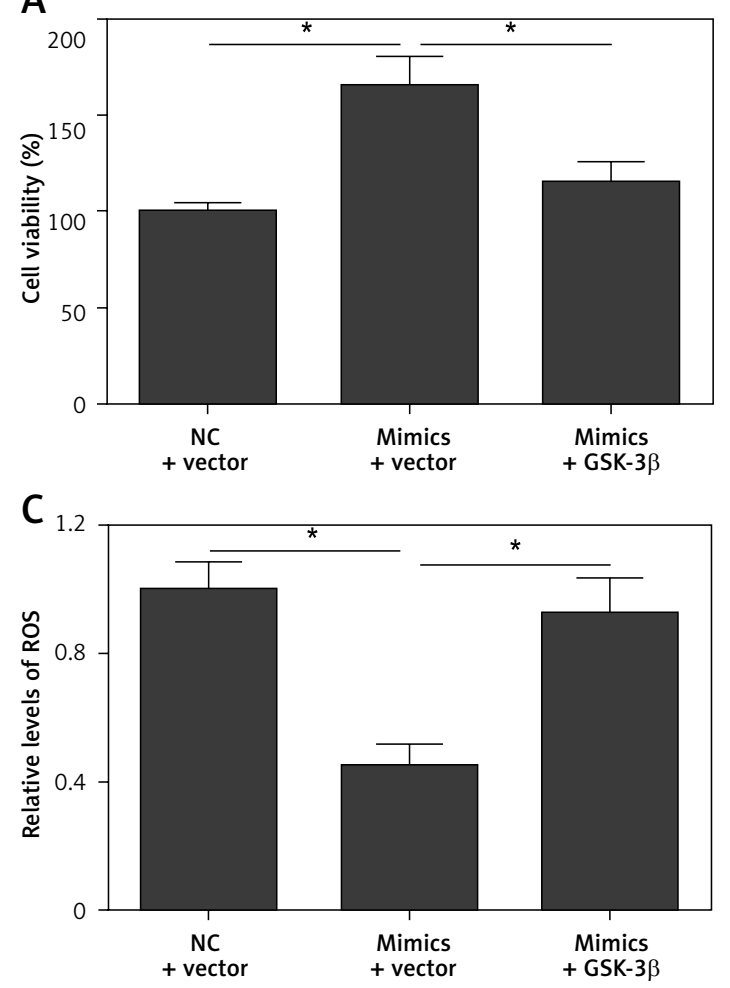

B

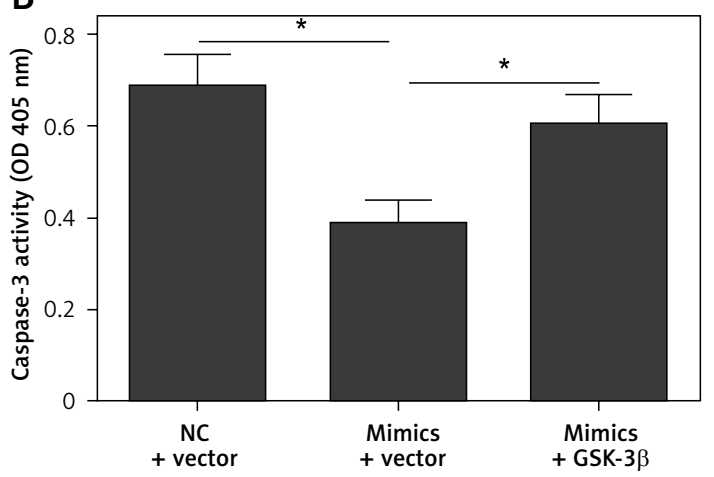

Figure 8. Restoration of GSK-3 $\beta$ expression reverses the protective effect of miR-135b-5p overexpression. HT22 cells were cotransfected with pCDNA3.1/GSK-3 $\beta$ vector and miR-135b-5p mimics for $24 \mathrm{~h}$ and then subjected to OGD/R treatment. A - Cell viability was measured by CCK- 8 assay. B - Cell apoptosis was detected by caspase-3 activity assay. C - ROS levels were measured by DCFHDA assay ${ }^{*} p<0.05$. 


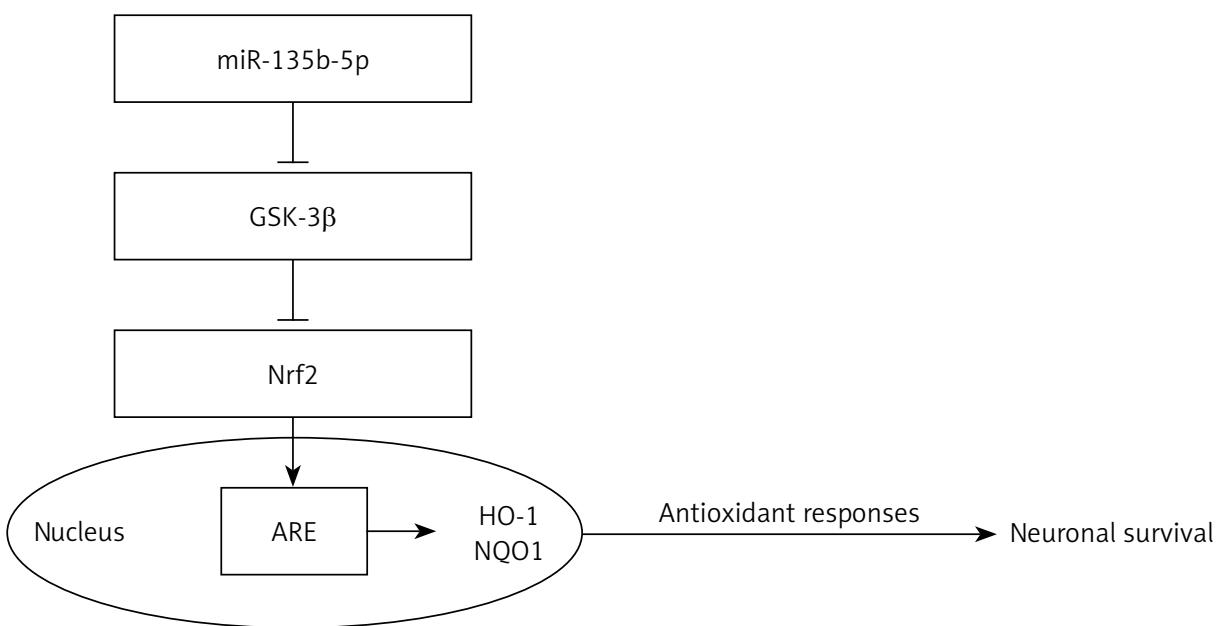

Figure 9. miR-135b-5p protects hippocampal neurons against OGD/R-induced injury by downregulation of GSK-3 $\beta$ and upregulation of Nrf2/ARE signaling pathway-mediated antioxidant responses

through downregulation of the Nrf2/ARE signaling pathway $[12,13]$. Therefore, inhibition of GSK-3 $\beta$ can promote activation of the Nrf2/ARE signaling pathway and alleviate neuronal damage [20]. Various neuroprotective drugs inhibit neuron apoptosis by targeting the GSK-3 $\beta / N r f 2 / A R E$ signaling pathway [34-37], suggesting that the GSK-3 $\beta$ / Nrf2/ARE signaling pathway plays an important role in regulating neuron apoptosis. In this study, we found that GSK-3 $\beta$ was a potential target gene of miR-135b-5p. We observed that overexpression of miR-135b-5p inhibits the expression of GSK-3 $\beta$ in neurons and protects neurons against OGD/R-induced injury by promoting activation of the Nrf2/ARE signaling pathway. Activation of the Nrf2/ARE signaling pathway can inhibit the inflammation and oxidative stress induced by ischemia and reperfusion [38, 39]. Research has shown that downregulation of the inflammatory response is beneficial to alleviate ischemia/ reperfusion injury [40]. Our results suggested that miR-135b-5p may serve as an inhibitor of GSK-3 $\beta$ which can thus activate the Nrf2/ARE signaling pathway, representing a novel molecular target for protecting neurons against OGD/R-induced injury.

A growing body of evidence has demonstrated that GSK-3 $\beta$ is regulated by various miRNAs, including miR-769 [41], miR-709 [42], and miR-26a [43]. In this study, we found that GSK-3 $\beta$ is also a target gene of miR-135b-5p. In line with our findings, several studies have shown that miR-135b-5p targets and regulates GSK-3 $\beta$ expression. Xiao et al. reported that miR-135b-5p promotes the radioresistance of glioblastoma multiforme cells by targeting GSK-3 $\beta$ [27]. miR-135b-5p mediates podocyte injury through targeting GSK-3 $\beta$ [44]. Our study further confirmed the functional relationship between miR-135b-5p and GSK-3 $\beta$.

Overall, our results show that overexpression of miR-135b-5p attenuates OGD/R-induced in- jury hippocampal neurons by downregulation of GSK-3 $\beta$ and upregulation of the Nrf2/ARE signaling pathway-mediated antioxidant responses (Figure 9). These results suggest a neuroprotective role for miR-135b-5p in OGD/R-induced injury. However, the precise role of miR-135b-5p in the modulation of neuronal survival by the GSK-3 $\beta$ / Nrf2/ARE signaling pathway in vivo requires further study using animal models.

\section{Acknowledgments}

This study was supported by the People's Liberation Army General Logistics Department Special Innovation Project (No. 16CXZO22), the International Science and Technology Cooperation Program of China (No. 2013DFA32610) and the National Natural Science Foundation of China (No. 81271450).

\section{Conflict of interest}

The authors declare no conflict of interest.

\section{References}

1. Feigin VL, Forouzanfar MH, Krishnamurthi R, et al. Global and regional burden of stroke during 1990-2010: findings from the Global Burden of Disease Study 2010. Lancet 2014; 383: 245-54.

2. Wechsler LR. Intravenous thrombolytic therapy for acute ischemic stroke. N Engl J Med 2011; 364: 2138-46.

3. Sims NR, Muyderman H. Mitochondria, oxidative metabolism and cell death in stroke. Biochim Biophys Acta 2010; 1802: 80-91.

4. Bartel DP. MicroRNAs: genomics, biogenesis, mechanism, and function. Cell 2004; 116: 281-97.

5. Huang Y, Shen XJ, Zou Q, et al. Biological functions of microRNAs: a review. J Physiol Biochem 2011; 67: 129-39.

6. Di Y, Lei Y, Yu F, et al. MicroRNAs expression and function in cerebral ischemia reperfusion injury. J Mol Neurosci 2014; 53: 242-50.

7. Ji Q, Gao J, Zheng Y, et al. Inhibition of microRNA-153 protects neurons against ischemia/reperfusion injury in 
an oxygen-glucose deprivation and reoxygenation cellular model by regulating Nrf2/HO-1 signaling. J Biochem Mol Toxicol 2017 in press.

8. Wang P, Liang X, Lu Y, et al. MicroRNA-93 downregulation ameliorates cerebral ischemic injury through the Nrf2/HO-1 defense pathway. Neurochem Res 2016; 41: 2627-35.

9. Seira O, Del Rio JA. Glycogen synthase kinase 3 beta (GSK3beta) at the tip of neuronal development and regeneration. Mol Neurobiol 2014; 49: 931-44.

10. Leroy K, Brion JP. Developmental expression and localization of glycogen synthase kinase-3beta in rat brain. J Chem Neuroanat 1999; 16: 279-93.

11. Woodgett JR. Recent advances in the protein kinase $B$ signaling pathway. Curr Opin Cell Biol 2005; 17: 150-7.

12. Li Y, Zhu J, Liu Y, et al. Glycogen synthase kinase 3beta influences injury following cerebral ischemia/reperfusion in rats. Int J Biol Sci 2016; 12: 518-31.

13. Chen X, Liu Y, Zhu J, et al. GSK-3beta downregulates Nrf2 in cultured cortical neurons and in a rat model of cerebral ischemia-reperfusion. Sci Rep 2016; 6: 20196.

14. Leng $Y$, Liang $M H$, Ren $M$, et al. Synergistic neuroprotective effects of lithium and valproic acid or other histone deacetylase inhibitors in neurons: roles of glycogen synthase kinase-3 inhibition. J Neurosci 2008; 28: 2576-88.

15. Chan K, Han XD, Kan YW. An important function of Nrf2 in combating oxidative stress: detoxification of acetaminophen. Proc Natl Acad Sci U S A 2001; 98: 4611-6.

16. Cuadrado A. Structural and functional characterization of Nrf2 degradation by glycogen synthase kinase 3/ beta-TrCP. Free Radic Biol Med 2015; 88: 147-57.

17. Satoh T, Okamoto SI, Cui J, et al. Activation of the Keap1/Nrf2 pathway for neuroprotection by electrophilic [correction of electrophillic] phase II inducers. Proc Natl Acad Sci U S A 2006; 103: 768-73.

18. Sandberg M, Patil J, D'Angelo B, et al. NRF2-regulation in brain health and disease: implication of cerebral inflammation. Neuropharmacology 2014; 79: 298-306.

19. Salazar M, Rojo Al, Velasco D, et al. Glycogen synthase kinase-3beta inhibits the xenobiotic and antioxidant cell response by direct phosphorylation and nuclear exclusion of the transcription factor Nrf2. J Biol Chem 2006; 281: 14841-51.

20. Pang T, Wang YJ, Gao YX, et al. A novel GSK-3beta inhibitor YQ138 prevents neuronal injury induced by glutamate and brain ischemia through activation of the Nrf2 signaling pathway. Acta Pharmacol Sin 2016; 37: 741-52.

21. He Y, Wang J, Yung VY, et al. MicroRNA-135b regulates apoptosis and chemoresistance in colorectal cancer by targeting large tumor suppressor kinase 2. Am J Cancer Res 2015; 5: 1382-95.

22. Li J, Liang $H$, Bai $M$, et al. miR-135b promotes cancer progression by targeting transforming growth factor beta receptor II (TGFBR2) in colorectal cancer. PLoS One 2015; 10: e0130194.

23. Zhang Y, Xing H, Guo S, et al. MicroRNA-135b has a neuroprotective role via targeting of beta-site APP-cleaving enzyme 1. Exp Ther Med 2016; 12: 809-14.

24. Schaller B, Graf R. Cerebral ischemia and reperfusion: the pathophysiologic concept as a basis for clinical therapy. J Cereb Blood Flow Metab 2004; 24: 351-71.

25. Li P, Fan JB, Gao Y, et al. miR-135b-5p inhibits LPS-induced TNFalpha production via silencing AMPK phosphatase Ppm1e. Oncotarget 2016; 7: 77978-86.

26. Song Z, Chen LL, Wang RF, et al. MicroRNA-135b inhibits odontoblast-like differentiation of human dental pulp cells by regulating Smad5 and Smad4. Int Endod J 2017; 50: 685-93.
27. Xiao S, Yang Z, Lv R, et al. miR-135b contributes to the radioresistance by targeting GSK3beta in human glioblastoma multiforme cells. PLoS One 2014; 9: e108810.

28. Valeri N, Braconi C, Gasparini P, et al. MicroRNA-135b promotes cancer progression by acting as a downstream effector of oncogenic pathways in colon cancer. Cancer Cell 2014; 25: 469-83.

29. Fan JB, Ruan JW, Liu W, et al. miR-135b expression downregulates Ppm1e to activate AMPK signaling and protect osteoblastic cells from dexamethasone. Oncotarget 2016; 7: 70613-22.

30. Hu K, Xie YY, Zhang C, et al. MicroRNA expression profile of the hippocampus in a rat model of temporal lobe epilepsy and miR-34a-targeted neuroprotection against hippocampal neurone cell apoptosis post-status epilepticus. BMC Neurosci 2012; 13: 115

31. Xu S, Zhang R, Niu J, et al. Oxidative stress mediated-alterations of the microRNA expression profile in mouse hippocampal neurons. Int J Mol Sci 2012; 13: 16945-60.

32. Cross DA, Culbert AA, Chalmers KA, et al. Selective small-molecule inhibitors of glycogen synthase kinase- 3 activity protect primary neurones from death. J Neurochem 2001; 77: 94-102.

33. Nonaka S, Chuang DM. Neuroprotective effects of chronic lithium on focal cerebral ischemia in rats. Neuroreport 1998; 9: 2081-4.

34. Wen X, Huang A, Hu J, et al. Neuroprotective effect of astaxanthin against glutamate-induced cytotoxicity in HT22 cells: involvement of the Akt/GSK-3beta pathway. Neuroscience 2015; 303: 558-68.

35. Li C, Pan Z, Xu T, et al. Puerarin induces the upregulation of glutathione levels and nuclear translocation of Nrf2 through PI3K/Akt/GSK-3beta signaling events in PC12 cells exposed to lead. Neurotoxicol Teratol 2014; 46: 1-9.

36. Zou Y, Hong B, Fan L, et al. Protective effect of puerarin against beta-amyloid-induced oxidative stress in neuronal cultures from rat hippocampus: involvement of the GSK-3beta/Nrf2 signaling pathway. Free Radic Res 2013; 47: 55-63.

37. Abdel-Aleem GA, Khaleel EF, Mostafa DG, et al. Neuroprotective effect of resveratrol against brain ischemia reperfusion injury in rats entails reduction of DJ-1 protein expression and activation of PI3K/Akt/GSK3b survival pathway. Arch Physiol Biochem 2016; 122: 200-13.

38. Sun G, Li Y, Ji Z. Atorvastatin attenuates inflammation and oxidative stress induced by ischemia/reperfusion in rat heart via the Nrf2 transcription factor. Int J Clin Exp Med 2015; 8: 14837-45.

39. Cheng L, Jin Z, Zhao R, et al. Resveratrol attenuates inflammation and oxidative stress induced by myocardial ischemia-reperfusion injury: role of Nrf2/ARE pathway. Int J Clin Exp Med 2015; 8: 10420-8.

40. Kim MS, Lee S, Jung N, et al. The vitamin D analogue paricalcitol attenuates hepatic ischemia/reperfusion injury through down-regulation of Toll-like receptor 4 signaling in rats. Arch Med Sci 2017; 13: 459-69.

41. Qiu HJ, Lu XH, Yang SS, et al. MiR-769 promoted cell proliferation in human melanoma by suppressing GSK3B expression. Biomed Pharmacother 2016; 82: 117-23.

42. Li M, Chen H, Chen L, et al. miR-709 modulates LPSinduced inflammatory response through targeting GSK-3beta. Int Immunopharmacol 2016; 36: 333-8.

43. Jiang JJ, Liu CM, Zhang BY, et al. MicroRNA-26a supports mammalian axon regeneration in vivo by suppressing GSK3beta expression. Cell Death Dis 2015; 6: e1865.

44. Yang $X$, Wang $X$, Nie F, et al. miR-135 family members mediate podocyte injury through the activation of Wnt/ beta-catenin signaling. Int J Mol Med 2015; 36: 669-77. 